\title{
Correlations of ultrasonic features with severity of liver cancer and p16 expression in patients with liver cancer
}

\author{
H. JIA, D. YAN, Q. XIAO*, G. ZHANG \\ Department of Ultrasound, Liaocheng People’s Hospital, Liaocheng, 252002, Shandong, China \\ *Correspondence: qingnaxiao@163.com
}

Received April 20, 2018 / Accepted June 26, 2018

\begin{abstract}
This paper analyzes the correlations of ultrasonic features with clinical-pathological manifestations and p16 expression in patients with liver cancer. A total of 84 patients with primary liver cancer were randomly enrolled. The characteristics of liver cancer were examined via conventional ultrasound and contrast-enhanced ultrasound (CEUS) before operation. The p16 protein expressions in liver cancer and para-carcinoma tissues were detected via immunohistochemistry (IHC), and the correlations of 16 positive expression with ultrasound parameters were also analyzed. It was manifested via ultrasound that $6.4 \%(3 / 47)$ of stage I-II liver cancer showed the equal-echo change in portal phase, while others faded and showed the equal-echo change with $32.4 \%$ (12/37) of stage III-IV liver cancer. There were no statistically significant differences in ultrasonic features of patients in stage I-II and stage III-IV in the arterial and delayed phases ( $\mathrm{p}>0.05)$. The positive expression rate of p16 protein in para-carcinoma tissues was $85.71 \%(72 / 84)$ which was significantly higher than that in liver cancer tissues $(30.95 \%, 26 / 84)(\mathrm{p}<0.05)$. Maximum intensity (IMAX), time to peak (TTP) and mean transit time (mTT) had no statistically significant differences between the p16 positive and negative groups, but there were statistically significant differences in rising slope $(\mathrm{RS})$ and washout time $(\mathrm{WT})(\mathrm{p}<0.05)$. Correlation analyses revealed that the positive expression of p16 had no significant correlations with IMAX, mTT and TTP ( $>0.05)$, but it positively correlated with RS (correlation coefficient $\mathrm{r}=0.377, \mathrm{p}<0.05)$ and negatively correlated with WT $(\mathrm{r}=-0.410, \mathrm{p}<0.05)$.

Conventional ultrasound and CEUS can evaluate the expression of p16 protein in liver cancer repeatedly and non-invasively. The evaluations therefore have great significance in clinical treatment of liver cancer.
\end{abstract}

Key words: liver cancer, ultrasound, contrast-enhanced ultrasound, p16

The mortality rate of liver cancer ranks third in global cancer and it is the second most common malignant tumor causing death in China; the vast majority of this is hepatocellular carcinoma $[1,2]$. The overall 5-year survival rate of liver cancer is less than 5\%, and there are 564,000 new cases and 549,000 deaths each year [3]. The main reason for its poor prognosis is that liver cancer easily invades the portal vein and leads to intrahepatic dissemination, thus resulting in high intrahepatic metastasis rate and high recurrence rate after liver cancer operation. The 5-year metastasis and recurrence rate is $61.5 \%$ after radical resection of liver cancer even in the early stage, and it is also up to $43.5 \%$ in small hepatocellular carcinoma [4]. Recurrence and metastasis after operation of liver cancer are not only important causes affecting its treatment effects, but also one of the most complex problems in the prevention and treatment of liver cancer. In addition to drug therapy, regular follow-up and close monitoring after liver cancer operation are important measures of early detection of recurrence and intrahepatic metastasis; and this is the key to improving the post-operative curative effect for liver cancer [5].

At present, the follow-up methods after operation of liver cancer include laboratory test, imaging examination and clinical observation. Contrast-enhanced ultrasound (CEUS), using the large difference in acoustic impedance between ultrasound contrast agent and body tissues, artificially increases the acoustic robustness between blood containing contrast agent and adjacent tissues. The ultrasound image contrast is therefore increased and it clearly displays the small vessel signal containing contrast agent and microvascular perfusion $[6,7]$. Compared with conventional ultrasound, CEUS has the advantages of sensitive manifestation of liver tumor, more accurate differential diagnosis and clear display of liver and tumor blood flow and tissue perfusion [8] 
P16, as a tumor suppressor, can inhibit cell proliferation through blocking the cell cycle; and its abnormal expression is also an important factor in tumor occurrence, development, invasion and metastasis [9].

Thorgeirsson et al analyzed the postoperative specimens of 383 patients with non-small cell lung cancer (NSCLC), and found that p16 inactivation exists in both lung squamous carcinoma and lung adenocarcinoma (84\% and 50\%) [10]. According to the meta-analysis of research data of 2,432 patients conducted by Zhang et al, hypermethylation of the p16 gene promoter in lung cancer is associated with poor patient prognosis [11]. Several previous studies have demonstrated the abnormal expression or function of p16 in liver cancer, but abnormal expression of p16 associated with liver cancer pathological features remains poorly understood.

This paper therefore summarizes the data of 84 outpatients and inpatients with primary liver cancer in our hospital from March 2014 to September 2016, and investigates correlations of liver CEUS with pathological features and p16 expression in patients with liver cancer.

\section{Patients and methods}

Clinical data. A total of 84 patients with primary liver cancer treated in the Oncology Department of our hospital from March 2014 to September 2016 were randomly enrolled; with 57 males and 27 females aged 33-78 years. Of these, 79 patients had a history of hepatitis B cirrhosis, 3 had hepatitis $C$ cirrhosis and 2 had hepatitis B complicated with hepatitis C. 55 patients received right liver tumor resection, 10 had middle lobectomy, 15 underwent left liver tumor resection and 4 had right hepatectomy. Other general clinical data is listed in Table 1. All patients received conventional

Table 1. General clinical data of patients enrolled.

\begin{tabular}{lcc}
\hline Item & $\mathbf{n}(\mathbf{8 4})$ & Percentage (\%) \\
\hline Age (years old) & & \\
$\quad<50$ & 36 & 42.86 \\
$\quad \geq 50$ & 48 & 57.14 \\
Sex & & \\
$\quad$ Male & 57 & 67.86 \\
$\quad$ Female & 27 & 32.14 \\
Tumor size (cm) & & \\
$\quad<5$ & 54 & 64.29 \\
$\quad \geq 5$ & 30 & 35.71 \\
Tumor-node-metastasis(TNM) staging & & \\
$\quad$ I-II & 47 & 55.95 \\
$\quad$ III-IV & 37 & 44.05 \\
Degree of differentiation & & \\
$\quad$ High differentiation & 17 & 20.24 \\
$\quad$ Moderate differentiation & 43 & 51.19 \\
$\quad$ Poor differentiation & 24 & 28.57 \\
Lymph node metastasis & & \\
$\quad$ Yes & 25 & 29.76 \\
$\quad$ No & 59 & 70.24 \\
\hline
\end{tabular}

ultrasound and liver CEUS before operation, and cancer tissues and para-carcinoma tissues were collected after the operation. All participants signed informed consent.

Ultrasonic examination. Philips-iU22 ultrasonic diagnostic instrument with the probe of $\mathrm{C} 5-2$ and frequency of $2.0-5.0 \mathrm{MHz}$ was used as the ultrasonic instrument, and PM/PI imaging software had the mechanical index of 0.04-0.08. The ultrasonic contrast agent SonoVue (Bracco) was the contrast agent, and phospholipid-coated sulfur hexafluoride $\left(\mathrm{SF}_{6}\right)$ the contrast micro-bubble. Before application, SonoVue was diluted with $5 \mathrm{ml}$ normal saline and mixed evenly, and $2.4 \mathrm{ml}$ SonoVue was extracted as the single dose. The patient was then injected with $2.4 \mathrm{ml}$ SonoVue by elbow intravenous bolus injection, rinsed with $5 \mathrm{ml}$ normal saline, and the whole process was observed in real time for 6 minutes. Enhancement of liver tumor in the arterial phase (10-30s), portal phase (30-120s) and delayed phase (121-360s) were observed and compared with the echo intensity of surrounding liver parenchyma (high, equal and low echo).

CEUS dynamic image data was analyzed quantitatively using SonoLiver CAP software developed by TomTec, and the time-intensity curve (TIC) was produced. CEUS parameters were maximum intensity (IMAX): the ratio of lesion region of interest (ROI) to reference ROI under the highest perfusion intensity. Time to peak (TTP) was the time from the start to IMAX of the contrast agent. Mean transit time (mTT): the time from the start to the time when TIC drops to half of IMAX. Rising slope (RS): IMAX/TTP. Washout time (WT) was the transformation time of the contrast agent from IMAX to equal enhancement.

Detection of $\mathrm{p} 16$ protein level by immunohistochemistry (IHC). Streptavidin-peroxidase (S-P) staining was performed in IHC: After dewaxing with xylene, dehydration with ethanol in gradient concentration, and antigen retrieval with sodium citrate buffer via microwave, peroxidase was blocked via $3 \% \mathrm{H}_{2} \mathrm{O}_{2}$ and tissues were sealed with $10 \%$ donkey serum. Phosphate buffered saline (PBS) was added as the negative control, and the primary antibody (p16, Abcam, USA, diluted at 1:200) was added drop-wise for incubation in a wet box at $4^{\circ} \mathrm{C}$ overnight. Tissues were washed with PBS 3 times the following day and incubated with ready-to-use universal secondary antibody (diluted at 1:2000), followed by color development via diaminobenzidine (DAB) and photography under the microscope. The brown and tan nuclei under the microscope indicated positive cells and these were counted. Positive cell count/total cell count in the field of view $>10 \%$ indicated positive expression.

Statistical methods. Data was statistically analyzed by Statistical Product and Service Solutions (SPSS) 10.0 software package. Measurement data was presented as mean \pm standard deviation $(\bar{\chi} \pm s)$. Student's t-test was used to compare the differences in indexes between the two groups and Spearman's rank correlation analysis correlated the two indices for abnormal distribution. $\mathrm{p}<0.05$ was considered statistically significant. 


\section{Results}

Ultrasonic features of liver cancer. CEUS examination of normal liver shows that hepatic arterial enhancement begins at approximately 10-20 seconds after injection and lasts approximately 10-15 seconds, while the portal venous phase begins after the arterial phase (30-45 seconds after injection) and lasts approximately two minutes. The delayed imaging then begins after the portal venous phase and lasts up to 6 minutes after injection. In liver cancer, however, the CEUS enhancement features were rapid enhancement in the arterial phase and decline in enhancement and low-echo change in the portal and delayed phases (Figure 1).

Correlations of ultrasound features with pathological grading of liver cancer. Ultrasound indicated that $80.85 \%$ (38/47) of stage I-II liver cancer had rapid enhancement and high echo in the arterial phase, while $89.19 \%(33 / 37)$ of stage III-IV liver cancer showed high echo; and there was no statistically significant difference in the manifestation in the arterial phase between the two groups ( $p>0.05)$. It was also demonstrated that $6.4 \%$ (3/47) of stage I-II liver cancer displayed the equal-echo change in the portal phase, while others faded and showed the low-echo change. Further, $32.4 \%(12 / 37)$ of stage III-IV liver cancer displayed the equal-echo change in the portal phase, and the difference was statistically significant $(\mathrm{p}<0.05)$. In contrast, $93.6 \%(44 / 47)$ of stage I-II liver cancer and 91.9\% (34/37) of stage III-IV liver cancer showed the low-echo change in the delayed phase, and there was no statistically significant difference $(\mathrm{p}>0.05)$ (Table 2).

IHC detection of p16 protein expressions in liver cancer tissues and para-carcinoma tissues. While P16 protein can be expressed in the cytoplasm and nucleus, the expression in para-carcinoma tissues and liver cancer tissues were mainly in the cytoplasm (Figure 2). The positive rate of p16 protein was $85.71 \%(72 / 84)$ in para-carcinoma tissues and $30.95 \%$
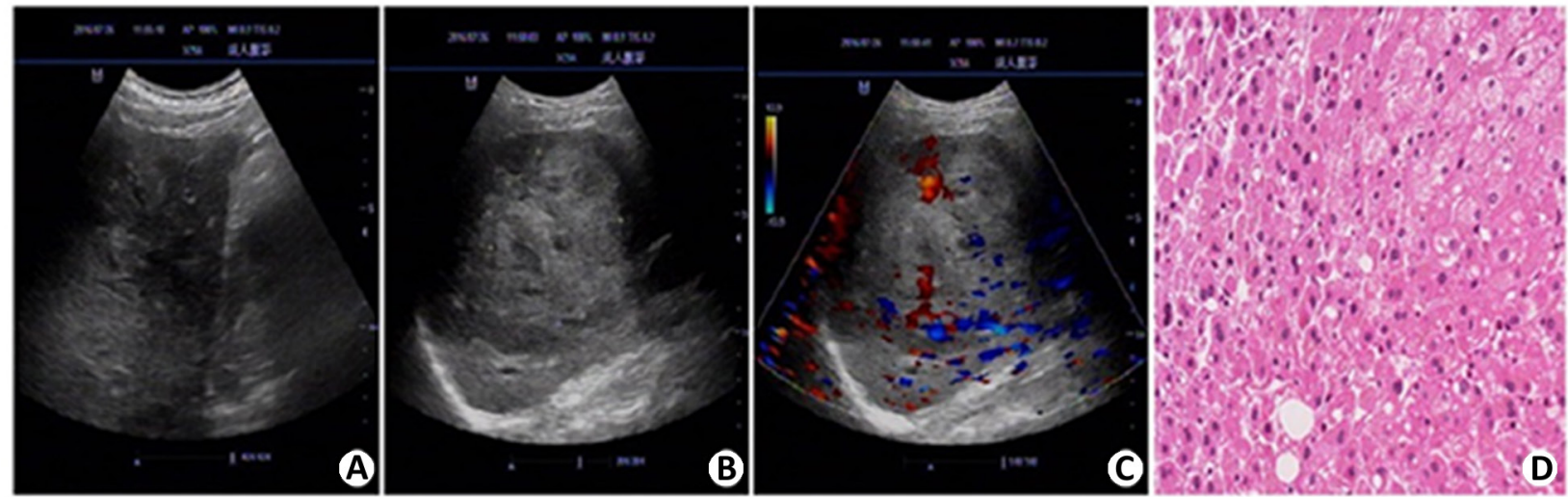

Figure 1. Ultrasound images and pathological features of patients with liver cancer. A male patient aged 44 years old. Ultrasound displays the incomplete liver capsule, heterogeneous echo in liver parenchyma, thickening, and multiple low echoes with regular shape and clear border within the liver, showing dispersed distribution. There is significant enhancement in the arterial phase and decline in enhancement in the portal phase and delayed phase.
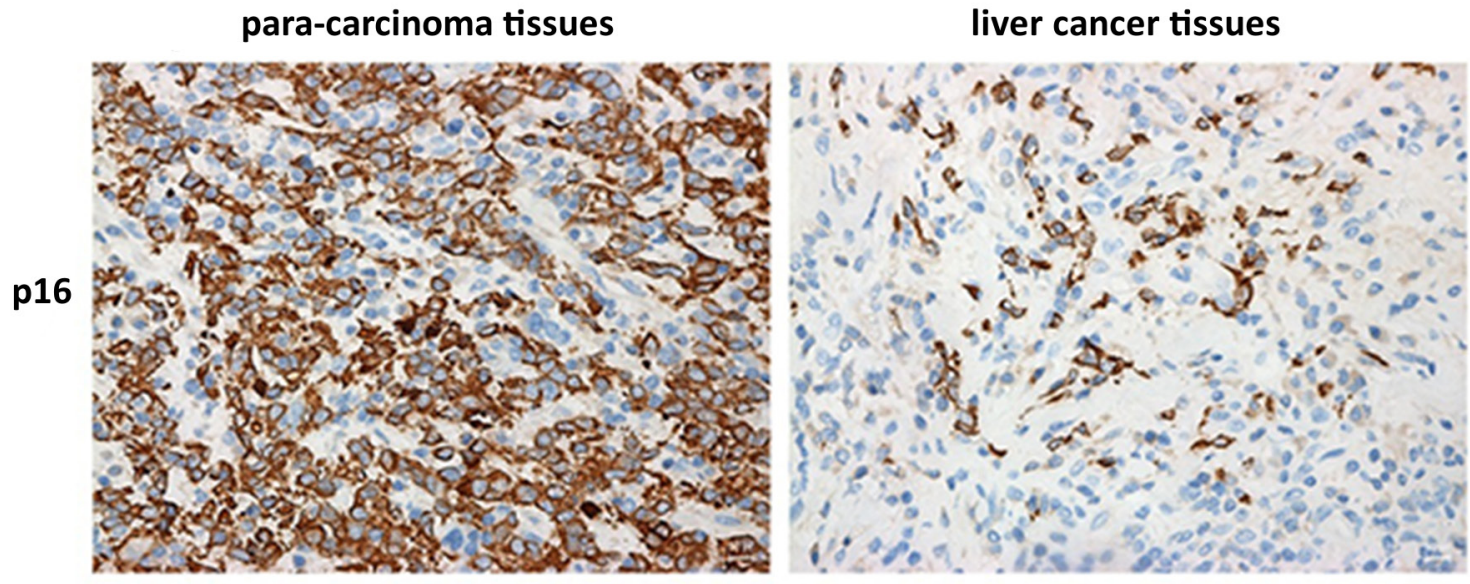

Figure 2. Detection of p16 protein expressions in liver cancer tissues and para-carcinoma tissues via IHC. 
Table 2. Pathological grading of liver cancer and manifestations in each phase of CEUS (n).

\begin{tabular}{|c|c|c|c|c|c|c|c|c|c|c|}
\hline \multirow{2}{*}{ Group } & \multicolumn{3}{|c|}{ Arterial phase } & \multicolumn{3}{|c|}{ Portal phase } & \multicolumn{3}{|c|}{ Delayed phase } & \multirow[b]{2}{*}{ Total } \\
\hline & High echo & Equal echo & Low echo & High echo & Equal echo & Low echo & High echo & Equal echo & Low echo & \\
\hline I-II & 38 & 6 & 3 & 0 & 3 & 44 & 0 & 3 & 44 & 47 \\
\hline III-IV & 33 & 3 & 1 & 0 & $12^{*}$ & $25^{\star}$ & 0 & 3 & 34 & 37 \\
\hline Total & 71 & 9 & 4 & 0 & 15 & 69 & 0 & 6 & 78 & 84 \\
\hline
\end{tabular}

Note: $\chi^{2}$ test for statistical analysis. ${ }^{*} \mathrm{p}<0.05$ vs. stage I-II.
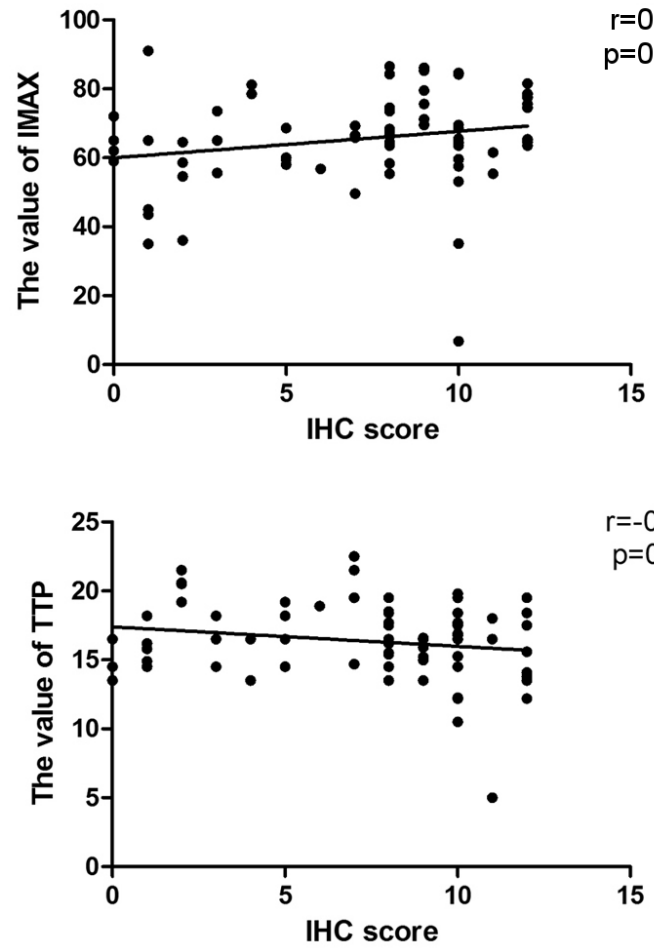
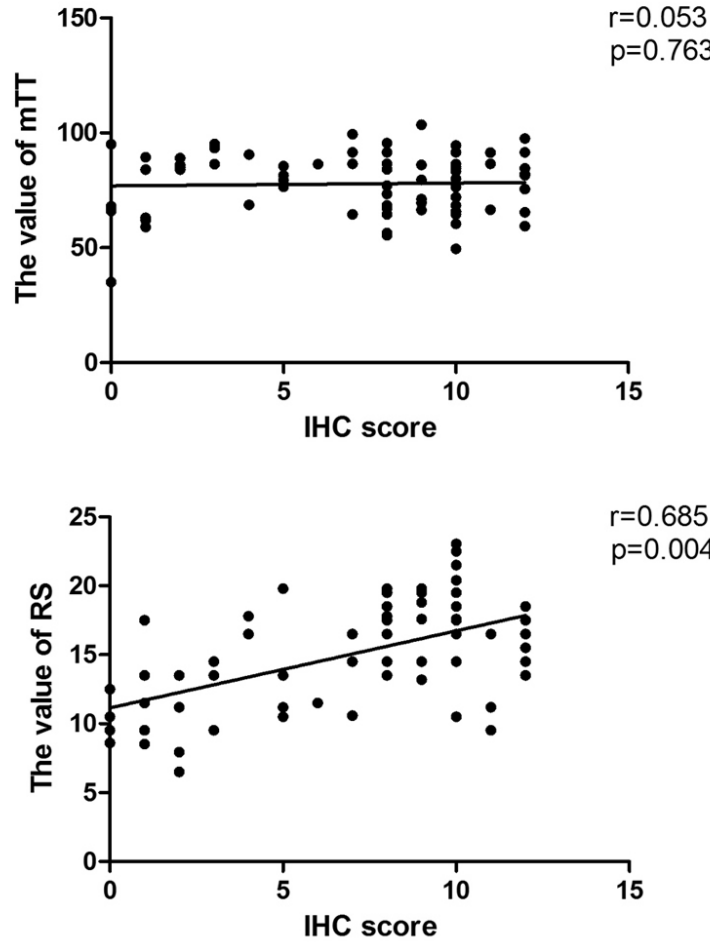

$r=0.685$

$p=0.004$

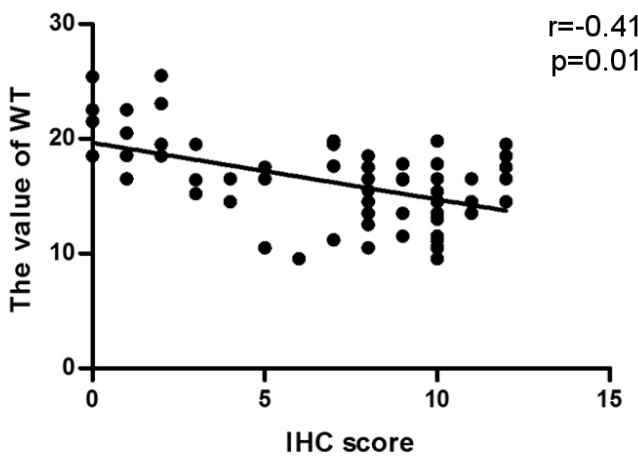

Figure 3. Correlation analyses of p16 positive expression with CEUS parameters.

Table 3. Comparison of p16 protein expression in liver cancer tissues and para-carcinoma tissues.

\begin{tabular}{lccccc}
\hline \multirow{2}{*}{ Item } & \multirow{n}{*}{$\mathbf{n}$} & \multicolumn{3}{c}{ P16 protein } & \multirow{2}{*}{ p-value } \\
\cline { 3 - 5 } & & - & + & Positive rate & \\
\hline Para-carcinoma tissues & 84 & 12 & 72 & $85.71 \%$ & $<0.05$ \\
Liver cancer tissues & 84 & 58 & 26 & $30.95 \%$ & $<0.05$ \\
\hline
\end{tabular}

$(26 / 84)$ in liver cancer tissues, and this difference was statistically significant $(\mathrm{p}<0.05$, Table 3$)$.

Correlations of $\mathrm{p} 16$ expression with CEUS parameters. CEUS parameters showed that IMAX, TTP and mTT had no statistically significant differences between the p16 positive and negative groups, but statistically significant differences 
Table 4. Correlations of p16 expression with CEUS parameters.

\begin{tabular}{lcccccc}
\hline P16 & n & IMAX & mTT & TTP & RS & WT \\
\hline Negative & 26 & $0.91 \pm 0.05$ & $80.9 \pm 20.7$ & $15.3 \pm 3.6$ & $17.1 \pm 4.4$ & $20.2 \pm 5.3$ \\
Positive & 58 & $0.93 \pm 0.07$ & $82.3 \pm 17.5$ & $16.6 \pm 3.3$ & $10.3 \pm 3.7$ & $28.4 \pm 3.7$ \\
$\mathrm{t}$ & & 1.313 & 0.316 & 1.134 & 3.026 & 2.531 \\
p-value & & 0.193 & 0.845 & 0.273 & $\mathbf{0 . 0 0 5}$ & $\mathbf{0 . 0 2 5}$ \\
\hline
\end{tabular}

were noted in RS and WT $(\mathrm{p}<0.05$, Table 4$)$. The RS in the p16 positive group was greater than that in negative group, thus indicating that the contrast agent inflow velocity in the positive group is higher. In addition, WT in p16 positive group was shorter than that in negative group, showing that the outflow time in the positive group is shorter and the lesions rapidly exhibited low enhancement.

Correlation analyses of p16 positive expression with CEUS parameters. Correlation analyses revealed that the positive expression of $\mathrm{p} 16$ had no significant correlations with IMAX, mTT and TTP ( $>0.05)$, but it positively correlated with RS (correlation coefficient $r=0.377, p<0.05$, Figure 3), suggesting that there is negative correlation of $\mathrm{p} 16$ positive expression with the contrast agent inflow velocity. The positive expression of p16 also negatively correlated with WT $(r=-0.410, p<0.05)$. This indicates that the longer contrast agent outflow time and slower fading equate with increasingly lower positive p16 expression rate (Figure 3).

\section{Discussion}

Conventional two-dimensional ultrasound and ultrasound Doppler examinations are the most commonly-used imaging methods in liver cancer operation follow-up. These have higher diagnostic value for changes in liver morphology and blood flow signals in liver cirrhosis through their $70 \%$ diagnosis and differential diagnosis accuracy for liver cirrhosis and space-occupying lesions [12]. However, certain damage and changes are caused in the intrahepatic anatomy, blood supply conditions and liver tissues of advanced liver cancer patients. Compared with patients with early liver cancer, the degree of liver cirrhosis is more serious in patients with recurrent liver cancer, and there is therefore increased difficulty in conventional ultrasound identifying small intrahepatic lesions. Conventional ultrasound is also not conducive to detecting blood flow signals in the lesion, and this gives significant decline in accuracy [13].

Ultrasonic contrast agent is a micron-sized pure blood pool contrast agent which reflects the tumor microvascular distribution and hemodynamics, and studies indicate that this remarkably improves diagnostic accuracy in small liver cancer [14]. Development of the CEUS quantitative analysis technique provides relevant ultrasonic parameters using quantitative software to accurately reflect tumor lesion microvascular perfusion and enhancement [15]. The CEUS enhancement features for liver cancer are the rapid improve- ment in arterial phase and declined enhancement and low-echo change in the portal and delayed phases [16]. This enables accurate tumor diagnosis and evaluation.

This study compared hemodynamic characteristics in patients with stage I-II liver cancer and those with stage III-IV liver cancer, and it revealed rapid enhancement in the arterial phase in both groups. The hepatic arterial blood supply was also dominant in stage III-IV liver cancer with dilation and tortuous artery supply, with abundant contrast agent rapid entrance. Low-echo change mainly dominated the portal and delayed phases, and paraplastic blood vessels and arteriovenous anastomotic branches were evident in the liver lesions.

The P16 tumor suppressor gene is a member of the cyclindependent kinase inhibitor (CDKI) family and was first discovered in double-hybrid yeast screening [17]. The p16 protein has156 amino acids containing 4 kankyrin repetitive sequences required for protein-protein interaction. These kankyrin repetitive sequences form a concave I-domain that binds to the non-catalytic aspect of human CDK4/CDK6, thus inhibiting the catalytic activity of the CDK4-6/cyclinD enzyme complex. The pRb activation is also inhibited, and the $\mathrm{G} 1$ to $\mathrm{S}$ cell-cycle phase is regulated via phosphorylation. Therefore, p16 is an important tumor suppressor gene [18].

Studies have also revealed that there are p16 point mutations and subsequent inactivation due to gene deletion in a variety of human tumors. For example, a specific p16 germline mutation is confirmed in familial melanoma and pancreatic cancer [19]. Recent studies have also shown that the aberrant methylation of p16 promoter is a major cause and this includes aberrant methylation of $59 \mathrm{CpG}$ islands in the p16 promoter region [20].

Herein, the expressions of p16 protein in 84 cancer and para-carcinoma tissues were detected via IHC. Results demonstrated that the p16 expression in these tissues was mainly located in the cytoplasm. The positive rate of p16 protein was $85.71 \%(72 / 84)$ in para-carcinoma tissues and $30.95 \%(26 / 84)$ in liver cancer tissues; with statistically significant difference at $\mathrm{p}<0.05$.

Correlations between p16 positive expression and CEUS parameters were then analyzed. This established that the ultrasonic modes in p16 positive group and negative group showed "fast-in and fast-out" features, the inflow velocity was higher and low enhancement appeared sooner because the inactivated p16 gene influenced tumor tissue growth through a variety of mechanisms. This leads to increased tumor malig- 
nancy; tumor micro-vessel density is increased, the number of arteriovenous fistula and hepatic arterial blood supply is increased, the portal vein blood supply is decreased and the circulation is hyper-dynamic. This combination provides more rapid contrast agent inflow and outflow, increased RS and shorter WT.

Portal vein blood supply in p16 negative patients was dominant and there were abundant blood sinuses in the tumor tissues. The contrast micro-bubbles had clear edges; so the contrast agent inflow velocity was lower and the outflow time was longer.

In conclusion, this study established that conventional ultrasound and liver CEUS can successfully evaluate p16 protein expression in liver cancer; repeatedly and non-invasively. We therefore provide greater understanding of both the severity of liver cancer and advances in its prognosis. This has great significance for liver cancer clinical treatment.

\section{References}

[1] CONTI F, BUONFIGLIOLI F, SCUTERI A, CRESPI C, BOLONDI $L$ et al. Early occurrence and recurrence of hepatocellular carcinoma in HCV-related cirrhosis treated with direct-acting antivirals. J Hepatol 2016; 65: 727-733. https:// doi.org/10.1016/j.jhep.2016.06.015

[2] BRUIX J, REIG M, SHERMAN M. Evidence-based diagnosis, staging, and treatment of patients with hepatocellular carcinoma. Gastroenterology 2016; 150: 835-853. https:// doi.org/10.1053/j.gastro.2015.12.041

[3] LIOVET JM, VILLANUEVAA, LACHENMAYER A, FINN RS. Advances in targeted therapies for hepatocellular carcinoma in the genomic era. Nat Rev Clin Oncol 2015; 12: 408-424. https://doi.org/10.1038/nrclinonc.2015.103

[4] NJEI B, ROTMAN Y, DITAH I, LIM JK. Emerging trends in hepatocellular carcinoma incidence and mortality. Hepatology 2015; 61: 191-199. https://doi.org/10.1002/hep.27388

[5] AYUSO C, RIMOLA J, VILANA R, BURREL M, DARNELL A et al. Diagnosis and staging of hepatocellular carcinoma (HCC): current guidelines. Eur J Radiol 2018; 101: 72-81. https://doi.org/10.1016/j.ejrad.2018.01.025

[6] KOSUGI C, FURUSE J, ISHII H, MARU Y, YOSHINO M et al. Needle tract implantation of hepatocellular carcinoma and pancreatic carcinoma after ultrasound-guided percutaneous puncture: clinical and pathologic characteristics and the treatment of needle tract implantation. World J Surg 2004; 28: 29-32. https://doi.org/10.1007/s00268-003-7003-y

[7] MAKUUCHI M, HASEGAWA H, YAMAZAKI S, TAKAYASU K, MORIYAMA N. The use of operative ultrasound as an aid to liver resection in patients with hepatocellular carcinoma. World J Surg 1987; 11: 615-621.
[8] VILANA R, FORNER A, BIANCHI L, GARCIA-CRIADO $A$, RIMOLA J et al. Intrahepatic peripheral cholangiocarcinoma in cirrhosis patients may display a vascular pattern similar to hepatocellular carcinoma on contrast-enhanced ultrasound. Hepatology 2010; 51: 2020-2029. https://doi. org/10.1002/hep. 23600

[9] BAEK MJ, PIAO Z, KIM NG, PARK C, SHIN EC et al. p16 is a major inactivation target in hepatocellular carcinoma. Cancer 2000; 89: 60-68.

[10] Thorgeirsson SS, Grisham JW. Molecular pathogenesis of human hepatocellular carcinoma. Nat Genet 2002; 31:339-346. https://doi.org/10.1038/ng0802-339

[11] LOU-QIAN Z, RONG Y, MING L, XIN Y, FENG J et al. The prognostic value of epigenetic silencing of p16 gene in NSCLC patients: a systematic review and meta-analysis. PLoS One 2013; 8: e54970. https://doi.org/10.1371/journal. pone.0054970

[12] SUGIHARA S, NAKASHIMA O, KOJIRO M, MAJIMA Y, TANAKA $M$ et al. The morphologic transition in hepatocellular carcinoma. A comparison of the individual histologic features disclosed by ultrasound-guided fine-needle biopsy with those of autopsy. Cancer 1992; 70: 1488-1492.

[13] YAMANAKA J, SAITO S, FUJIMOTO J. Impact of preoperative planning using virtual segmental volumetry on liver resection for hepatocellular carcinoma. World J Surg 2007; 31: 1251-1257. https://doi.org/10.1007/s00268-007-9020-8

[14] SQUIRES JH, BILL A, THIERET J, SQUIRES JE. Identification of Suspected Hepatocellular Carcinoma with ContrastEnhanced Ultrasound. J Pediatr 2017; 182: 398-398.e1. https://doi.org/10.1016/j.jpeds.2016.12.029

[15] LORIA F, LORIA G, BASILE S, CREA G, FROSINA L et al. Role of contrast-enhanced ultrasound in the evaluation of vascularization of hepatocellular carcinoma. Hepatoma Res 2016; 14: 210-227. https://doi.org/10.20517/23945079.2016.27

[16] IKEDA A, OIWA Y, KOKURYU H. Contrast-enhanced ultrasonography evaluation of hepatocellular carcinoma with peritumoral fat-spared area: a case report. J Med Ultrason (2001) 2018; 45: 325-329. https://doi.org/10.1007/s10396017-0822-5

[17] QU Z, JIANG Y, LI H, YU DC, DING YT. Detecting abnormal methylation of tumor suppressor genes GSTP1, P16, RIZ1, and RASSF1A in hepatocellular carcinoma and its clinical significance. Oncol Lett 2015; 10: 2553-2558. https:// doi.org/10.3892/ol.2015.3536

[18] SUN G, ZHANG C, FENG M, LIU W, XIE H et al. Methylation analysis of p16, SLIT2, SCARA5, and Runx3 genes in hepatocellular carcinoma. Medicine (Baltimore) 2017; 96: e8279. https://doi.org/10.1097/MD.0000000000008279

[19] BARTSCH DK, SINA-FREY M, LANG S, WILD A, GERDES B et al. CDKN2A germline mutations in familial pancreatic cancer. Ann Surg 2002; 236: 730-737. https://doi. org/10.1097/01.SLA.0000036393.89509.4E

[20] Tang H, Yao X, Li C, Liu P, Ma B. Methylation of DAPK, P16 and E-Cadherin gene in liver cancer tissues and protein expression level. Int J Clin Exp Pathol 2016; 9: 3787-3792. 